\title{
Ações de controle da hanseníase na atenção primária à saúde: uma revisão integrativa
}

\author{
Thiáskara Ramile Caldas Leite ${ }^{\mathrm{a}^{*}}$, Ingrid Grangeiro Bringel Silva ${ }^{\mathrm{a}}$, Fernanda Moura \\ Lanza $^{\mathrm{b}}$, Evanira Rodrigues Maia ${ }^{\mathrm{a}}$, Maria do Socorro Vieira Lopes ${ }^{\mathrm{a}}$, Edilma Gomes \\ Rocha Cavalcante $^{\mathrm{a}}$
}

\author{
${ }^{\text {a}}$ Universidade Regional do Cariri - URCA, Crato, CE, Brasil \\ ${ }^{\mathrm{b}}$ Universidade Federal de São João del-Rei - UFSJ, São João del-Rei, MG, Brasil
}

Histórico do Artigo:
Recebido em
07/03/2020
Aceito em
18/07/2020

\section{Palavras-chave:}

Hanseníase; controle de doenças transmissíveis; atenção primária à saúde

\begin{abstract}
RESUMO
A hanseníase ainda é considerada um importante problema de saúde pública pela magnitude de pessoas que atinge e pelo alto poder incapacitante. O objetivo deste estudo foi identificar na literatura científica nacional e internacional as medidas utilizadas na Atenção Primária à Saúde para o controle da hanseníase. Trata-se de revisão integrativa da literatura onde foram avaliadas as publicações científicas nas bases de dados: Literatura Latino-Americana e do Caribe em Ciências da Saúde (LILACS), Medical Literature Analysis and Retrieval Sistem on-line (Medline), Cumulative Index to Nursing and Allied health Literature (CINAHL), na Base de Dados de Enfermagem (BDENF), Índice Bibliográfico Espanhol de Ciências da Saúde (IBECS) e no diretório de revistas Scientific Electronic Library Online (SciELO). Na busca inicial foram encontrados 1234 artigos e após aplicação dos critérios de inclusão, exclusão e leitura na íntegra, selecionou-se 14 artigos, publicados entre 2006 e 2017. Identificou-se que as medidas utilizadas na Atenção Primária à Saúde para o controle da hanseníase foram: capacitação profissional, descentralização das atividades, atividades de educação em saúde, definição de atribuições profissionais e uso de instrumentos específicos na assistência de Enfermagem. Conclui-se que essas medidas de prevenção e controle estão em consonância com as recomendações mundiais e do governo brasileiro para a redução da carga da hanseníase, evidenciando os esforços para a descentralização das ações na Atenção Primária à Saúde.
\end{abstract}

\section{Actions to control attention in health care: an integrative review}

\section{ABSTRACT}

Leprosy is still considered an important public health problem due to the magnitude of people it affects and the high disabling power. The aim of this study was to identify in the national and international scientific literature the measures used in Primary Health Care to control leprosy. This is an integrative literature review where scientific publications in the databases were evaluated: Latin American and Caribbean Literature in Health Sciences (LILACS), Medical Literature Analysis and Retrieval System on-line (Medline), Cumulative Index to Nursing and Allied health Literature (CINAHL), in the Nursing Database (BDENF), Spanish Bibliographic Index of Health Sciences (IBECS) and in the directory of Scientific Electronic Library Online magazines (SciELO). In the initial search, 1234 articles were found and after applying the inclusion, exclusion and reading criteria in full, 14 articles were selected, published between 2006 and 2017. It was identified that the measures used in Primary Health Care for the control of leprosy were: professional training, decentralization of activities, health education activities, definition of professional duties and use of specific instruments in nursing care. It is concluded that these prevention and control measures are in line with the global and Brazilian government recommendations for the reduction of the leprosy burden, evidencing the efforts to decentralize the actions in primary health care.

\section{Introdução}

A hanseníase ainda é um importante problema de saúde pública pela magnitude de pessoas que atinge e pelo alto poder incapacitante (1). Nos últimos anos, mostra um decréscimo de casos em nível mundial (em 2009 foram notificados 244.796 casos,

\footnotetext{
* Autor correspondente: thiaskaracaldas@ gmail.com (Leite T.R.C.)
} 
enquanto em 2018 registrou-se 208.619 casos), mas a sua eliminação em alguns países ainda é desafiadora (2).

O Brasil está entre os três países que apresentaram aumento do número de casos novos ao comparar a situação epidemiológica de 2017 e 2018 (2), o que pode estar associado as disparidades regionais pela grande extensão territorial do país e pelas desigualdades socioeconômicas (3). Deve-se considerar também que as campanhas de detecção de casos e as avaliações de contatos podem estar sendo mais efetivas, sugerindo uma maior eficiência dos serviços de saúde (3).

Embora haja engajamento do governo brasileiro para reduzir a carga da doença $(4,5)$, a maioria dos casos ainda apresentam algum grau de incapacidade física no momento do diagnóstico (2), o que sugere fragilidades dos serviços de saúde em realizar o diagnóstico oportuno (6).

A persistência da taxa de detecção em menores de 15 anos e a ocorrência de municípios silenciosos (7) são situações preocupantes que reforçam a necessidade de implementar os pilares da Estratégia Global para Hanseníase: o fortalecimento do controle, da coordenação e da parceria governamental, o combate da hanseníase e suas complicações, e o enfrentamento da discriminação com promoção da inclusão social (1).

A implementação de ações no âmbito da Atenção Primária à Saúde (APS) é considerada a melhor estratégia para o controle da hanseníase, por facilitar a divulgação dos sinais e sintomas para a comunidade, o acesso ao diagnóstico oportuno e o tratamento até a cura, na perspectiva de prevenir as incapacidades reduzindo a exclusão social e o estigma relacionados à doença, além de uma abordagem sistemática e qualificada para o desenvolvimento das ações de vigilância dos contatos domiciliares e sociais (4).

Um estudo realizado em Ruanda, localizada na África Oriental apontou que a sensibilização dos agentes comunitários de saúde, vigilância ativa da doença, coleta de dados e vontade política foram estratégias efetivas, pois houve diminuição das taxas de prevalência e incidência no período de 1995 a 2011 (8).

As orientações atuais dos planos de enfrentamento sugerem que as atividades devem ser desenvolvidas em cada ponto da rede de atenção, utilizando-se do apoio dos serviços da média e alta complexidade para obter a integralidade da atenção à pessoa com hanseníase e seus familiares (5) com as devidas definições de responsabilidades, pois são importantes para a redução da carga da doença.

Entretanto, apesar das recomendações para ações de eliminação da hanseníase como problema de saúde pública (4), observa-se que ainda há dificuldades na integração das ações para controle da hanseníase nos serviços de APS (6). Os estudos mostram que os principais motivos são: número reduzido de profissionais capacitados (9), alta rotatividade de profissionais (10), falta de instrumentos para realização do exame dermatoneurológico e baciloscópico até a ausência de recursos de divulgação dos sinais e sintomas (11) e baixa adesão às medidas de vigilância à saúde, como busca ativa e exames de contatos (12).

Nesse sentido, torna-se oportuno compreender e discutir as ações de controle da hanseníase que estão sendo implementadas nos serviços de APS no Brasil e no mundo para a redução da carga da doença. Assim, o objetivo deste estudo foi identificar na literatura científica nacional e internacional as medidas utilizadas na Atenção Primária à Saúde para o controle da hanseníase.

\section{Materiais e métodos}

Revisão integrativa que seguiu o trajeto metodológico em seis etapas: elaboração da questão da pesquisa, amostragem ou busca na literatura, categorização dos estudos, 
avaliação dos estudos incluídos na revisão, interpretação dos resultados e apresentação da revisão (13).

Na primeira etapa, formulou-se a questão norteadora: "Quais medidas de controle da hanseníase estão sendo utilizadas na atenção primária à saúde no contexto nacional e internacional?"

A busca foi realizada no Portal de Periódicos da Coordenação de Aperfeiçoamento de Pessoal de Nível Superior (CAPES) nas seguintes bases de dados: Literatura LatinoAmericana e do Caribe em Ciências da Saúde (LILACS), Medical Literature Analysis and Retrieval Sistem on-line (Medline), Cumulative Index to Nursing and Allied health Literature (CINAHL), na Base de Dados de Enfermagem (BDENF), Índice Bibliográfico Espanhol de Ciências da Saúde (IBECS) e no diretório de revistas Scientific Electronic Library Online (SciELO).

A coleta de dados ocorreu nos meses de setembro e outubro de 2017 e em todas as bases de dados utilizou-se a busca avançada com os descritores localizados no Medical Subject Headings (MeSH) "Primary Health Care", "Communicable Disease Control" e "Leprosy" nos idiomas inglês, português e espanhol. Realizou-se a adequação desses descritores com as particularidades das bases de dados: Descritores de Ciências da Saúde (DeCS) e Títulos Cinahl.

Os artigos científicos encontrados na busca foram filtrados de acordo com os seguintes critérios de inclusão: texto completo disponível em inglês, português e/ou espanhol na modalidade artigo original, com ênfase nas medidas de controle para hanseníase na atenção primária, com a delimitação de tempo de 2000 a 2017. Optou-se por este recorte temporal, pois no ano 2000 foi alcançada a eliminação da hanseníase como problema de saúde pública em nível mundial (1). Na busca inicial foram encontrados 1234 artigos.

Os critérios de exclusão foram: editoriais, comentários, comunicações breves, artigos de reflexão, revisões narrativas, revisões sistemáticas, documentários, ensaios, resumos, teses, dissertações, resenhas e relatos de experiência; publicações duplicadas e artigos que não tivessem enfoque no assunto abordado. Destes, 956 foram eliminados após leitura dos títulos e resumos.

A síntese dos resultados obtidos nas etapas de busca dos artigos está apresentada no Quadro 1.

Quadro 1 - Estudos primários incluídos na revisão integrativa por meio de cruzamentos dos MeSH Terms de acordo com as bases de dados.

\begin{tabular}{|c|c|c|c|c|c|}
\hline Artigos selecionados na busca primária para leitura de título e resumo \\
\hline LILACS & BDENF & IBECS & SCIELO & CINHAL & MEDLINE \\
\hline 88 & 18 & 31 & 41 & 74 & 982 \\
\hline \multicolumn{7}{|c|}{ TOTAL: 1234} \\
\hline \multicolumn{7}{|c|}{ Avaliação da disponibilidade de textos completos } \\
\hline LILACS & BDENF & IBECS & SCIELO & CINHAL & MEDLINE \\
\hline 39 & 11 & 4 & 41 & 21 & 162 \\
\hline \multicolumn{7}{|c|}{ TOTAL: 278} \\
\hline \multicolumn{7}{|c|}{ Avaliação dos critérios de inclusão e exclusão } \\
\hline LILACS & BDENF & IBECS & SCIELO & CINHAL & MEDLINE \\
\hline 16 & 5 & 0 & 8 & 4 & 11 \\
\hline \multicolumn{7}{|c|}{ TOTAL: 44 } \\
\hline \multicolumn{7}{|c|}{ Incluídos após leitura na íntegra } \\
\hline LILACS & BDENF & IBECS & SCIELO & CINHAL & MEDLINE \\
\hline 6 & 2 & 0 & 3 & 3 & 3 \\
\hline 6
\end{tabular}


A definição das informações a serem extraídas dos estudos selecionados ocorreu na terceira fase. Os dados foram analisados conforme: ano, objetivo, desenho, além das medidas utilizadas para o controle da hanseníase na Atenção Primária à Saúde.

Posterior a essa classificação, realizou-se avaliação e análise crítica com o propósito de verificar a contribuição de cada estudo para responder à questão norteadora. Realizou-se na quinta fase, a síntese e discussão dos achados, identificando principais resultados e conclusões. Na sexta fase desta revisão, os resultados foram apresentados em quadros e classificados por categorias.

\section{Resultados}

Para uma melhor compreensão dos resultados, a caracterização dos estudos incluídos na revisão integrativa está apresentada na Tabela 1. Os artigos foram denominados de A1 a A14.

Tabela 1 - Características dos estudos incluídos na revisão integrativa.

\begin{tabular}{|c|c|c|c|}
\hline $\begin{array}{c}\text { Estudo/A } \\
\text { no }\end{array}$ & Objetivo & Desenho & $\begin{array}{l}\text { Medidas utilizadas na Atenção Primária } \\
\text { à Saúde para o controle da hanseníase }\end{array}$ \\
\hline $\begin{array}{l}\text { A1 (14)/ } \\
2006\end{array}$ & $\begin{array}{l}\text { Avaliar o progresso da integração } \\
\text { do controle da hanseníase no } \\
\text { sistema geral de saúde usando } \\
\text { categorias definidas. }\end{array}$ & $\begin{array}{l}\text { Estudo descritivo } \\
\text { exploratório com } \\
\text { delineamento } \\
\text { transversal }\end{array}$ & $\begin{array}{c}\text { Criação de Núcleos de Hanseníase na sede } \\
\text { dos distritos para fornecer suporte de } \\
\text { referência. }\end{array}$ \\
\hline $\begin{array}{c}\mathrm{A} 2(15) / \\
2007\end{array}$ & $\begin{array}{l}\text { Descrever as estratégias de } \\
\text { eliminação realizadas em Duque } \\
\text { de Caxias, município do Estado } \\
\text { do Rio de Janeiro, Brasil. }\end{array}$ & $\begin{array}{l}\text { Estudo descritivo } \\
\text { exploratório com } \\
\text { delineamento } \\
\text { ecológico. }\end{array}$ & $\begin{array}{c}\text { Descentralização do atendimento ao } \\
\text { paciente; campanhas localizadas, } \\
\text { principalmente em bairros endêmicos, com } \\
\text { o intuito de educar a população sobre a } \\
\text { doença, rastrear casos novos, capacitar os } \\
\text { profissionais das unidades da APS, e o } \\
\text { incentivo à descentralização da assistência } \\
\text { para essas unidades. }\end{array}$ \\
\hline $\begin{array}{c}\mathrm{A} 3(16) / \\
2007\end{array}$ & $\begin{array}{c}\text { Avaliar o impacto de um } \\
\text { programa de treinamento sobre a } \\
\text { detecção da hanseníase no nível } \\
\text { de atenção primária. }\end{array}$ & $\begin{array}{l}\text { Estudo de intervenção } \\
\text { educativa. }\end{array}$ & $\begin{array}{l}\text { O treinamento de um dia em dermatologia } \\
\text { básica mostrou uma grande melhora na } \\
\text { habilidade dos profissionais da APS no } \\
\text { atendimento dos usuários com hanseníase. }\end{array}$ \\
\hline $\begin{array}{l}\text { A4 (17)/ } \\
2008\end{array}$ & $\begin{array}{c}\text { Identificar e caracterizar a } \\
\text { implementação do Programa de } \\
\text { Controle da Hanseníase em serviços } \\
\text { de saúde de Bauru tendo como } \\
\text { referência o controle da doença. }\end{array}$ & $\begin{array}{l}\text { Estudo descritivo } \\
\text { exploratório com } \\
\text { delineamento } \\
\text { transversal. }\end{array}$ & $\begin{array}{c}\text { Atividades de acolhimento/suspeição } \\
\text { diagnóstica, busca ativa de casos, } \\
\text { educação em saúde e vacinação dos } \\
\text { contatos. }\end{array}$ \\
\hline $\begin{array}{l}\text { A5 }(18) / \\
2008\end{array}$ & $\begin{array}{l}\text { Avaliar a perspectiva de } \\
\text { eliminação da hanseníase no } \\
\text { Estado do Paraná. }\end{array}$ & $\begin{array}{l}\text { Estudo descritivo } \\
\text { exploratório com } \\
\text { delineamento } \\
\text { transversal. }\end{array}$ & $\begin{array}{c}\text { Descentralização das atividades de } \\
\text { controle da hanseníase para as unidades de } \\
\text { APS. }\end{array}$ \\
\hline $\begin{array}{l}\text { A6 (19)/ } \\
2009\end{array}$ & $\begin{array}{l}\text { Desenvolver um curso de educação } \\
\text { a distância em hanseníase para } \\
\text { treinamento de equipes das Equipes } \\
\text { de Saúde da Família. }\end{array}$ & $\begin{array}{l}\text { Estudo de intervenção } \\
\text { educativa. }\end{array}$ & $\begin{array}{l}\text { Treinamento de profissionais da APS por } \\
\text { meio de um modelo interativo de tele- } \\
\text { educação. } \\
\text { Houve melhoria nas habilidades de } \\
\text { diagnóstico de caso de hanseníase. }\end{array}$ \\
\hline $\begin{array}{l}\text { A7 (20)/ } \\
2009\end{array}$ & $\begin{array}{l}\text { Analisar o instrumento de } \\
\text { consulta de enfermagem utilizado } \\
\text { junto à clientela atendida no } \\
\text { Programa de Hanseníase de uma } \\
\text { Unidade de Atenção Primária à } \\
\text { Saúde e identificar as principais } \\
\text { necessidades de saúde e as ações } \\
\text { de enfermagem propostas. }\end{array}$ & $\begin{array}{l}\text { Estudo descritivo } \\
\text { exploratório com } \\
\text { delineamento } \\
\text { transversal. }\end{array}$ & $\begin{array}{l}\text { Aplicação de instrumento de consulta de } \\
\text { enfermagem denominados "Caso Novo" } \\
\text { (CN) e "Consulta de Seguimento" (CS), } \\
\text { que tiveram por base o Processo de } \\
\text { Enfermagem proposto por Wanda de } \\
\text { Aguiar Horta, mostraram-se adequados } \\
\text { para identificar as necessidades dos } \\
\text { usuários com hanseníase. Isso favoreceu } \\
\text { intervenções conjuntas com a equipe } \\
\text { multiprofissional, contribuindo para a } \\
\text { prevenção de incapacidades físicas, bem } \\
\text { como a realização de educação em saúde } \\
\text { para os pacientes e familiares. }\end{array}$ \\
\hline
\end{tabular}


Vittalle - Revista de Ciências da Saúde v. 32, n. 3 (2020) 175-186

Continuação

\begin{tabular}{|c|c|c|c|}
\hline $\begin{array}{c}\text { Estudo/A } \\
\text { no }\end{array}$ & Objetivo & Desenho & $\begin{array}{l}\text { Medidas utilizadas na Atenção Primária } \\
\text { à Saúde para o controle da hanseníase }\end{array}$ \\
\hline $\begin{array}{c}\text { A8 }(21) / \\
2011\end{array}$ & $\begin{array}{l}\text { Aumentar o conhecimento sobre a } \\
\text { hanseníase dos contatos intra- } \\
\text { domiciliares com histórico de } \\
\text { hanseníase. }\end{array}$ & $\begin{array}{l}\text { Estudo de intervenção } \\
\text { educativa. }\end{array}$ & $\begin{array}{c}\text { Intervenção educativa com familiares/ } \\
\text { cuidadores de pacientes com hanseníase } \\
\text { para melhorar o conhecimento sobre a } \\
\text { doença. }\end{array}$ \\
\hline $\begin{array}{l}\text { A9 }(22) / \\
2011\end{array}$ & $\begin{array}{l}\text { Conhecer a especificidade dos } \\
\text { serviços de saúde e quais } \\
\text { estratégias são empregadas por } \\
\text { esses serviços para fazer frente ao } \\
\text { processo de controle da } \\
\text { hanseníase como problema de } \\
\text { saúde pública. }\end{array}$ & $\begin{array}{l}\text { Estudo descritivo } \\
\text { exploratório de } \\
\text { abordagem qualitativa. }\end{array}$ & $\begin{array}{c}\text { Reuniões clínicas, capacitação de } \\
\text { odontólogos para suspeição diagnóstica, } \\
\text { supervisão das ações realizadas na } \\
\text { Estratégia de Saúde da Família por } \\
\text { equipes compostas por profissionais mais } \\
\text { experientes no manejo da hanseníase e a } \\
\text { ampliação da definição dos contatos } \\
\text { domiciliares. }\end{array}$ \\
\hline $\begin{array}{l}\mathrm{A} 10(23) / \\
2014\end{array}$ & $\begin{array}{c}\text { Analisar o estigma em hanseníase } \\
\text { sob a perspectiva de profissionais } \\
\text { da saúde e gestores e sua relação } \\
\text { com as ações de controle em } \\
\text { municípios da microrregião de } \\
\text { Araçuaí. }\end{array}$ & $\begin{array}{l}\text { Estudo descritivo } \\
\text { exploratório com } \\
\text { delineamento } \\
\text { transversal. }\end{array}$ & $\begin{array}{c}\text { Divulgação da hanseníase para a } \\
\text { comunidade por meio das orientações dos } \\
\text { Agentes Comunitários de Saúde (ACS) } \\
\text { durante as visitas domiciliares, } \\
\text { distribuição de panfletos, programas de } \\
\text { rádio, realização de grupos operativos e } \\
\text { sala de espera para a redução do estigma } \\
\text { em relação a doença. }\end{array}$ \\
\hline $\begin{array}{l}\text { A11 (10)/ } \\
2015\end{array}$ & $\begin{array}{l}\text { Analisar os fatores que } \\
\text { influenciam a integração das } \\
\text { ações de controle da hanseníase } \\
\text { na Atenção Primária à Saúde. }\end{array}$ & $\begin{array}{l}\text { Estudo descritivo } \\
\text { exploratório com } \\
\text { delineamento } \\
\text { transversal. }\end{array}$ & $\begin{array}{l}\text { Os ACS realizaram suspeição diagnóstica } \\
\text { durante a visita domiciliar, encaminham o } \\
\text { paciente em caso de reações, buscam os } \\
\text { contatos e os faltosos e, em situações } \\
\text { especiais, supervisionam o tratamento. O } \\
\text { profissional enfermeiro é o que está mais } \\
\text { envolvido com as atividades de } \\
\text { capacitação dos ACS. }\end{array}$ \\
\hline $\begin{array}{l}\mathrm{A} 12(24) / \\
2015\end{array}$ & $\begin{array}{c}\text { Avaliar o conhecimento e a } \\
\text { prática de enfermeiros da atenção } \\
\text { primária de saúde quanto às ações } \\
\text { de controle e eliminação da } \\
\text { hanseníase. }\end{array}$ & $\begin{array}{l}\text { Estudo avaliativo, de } \\
\text { natureza qualitativa. }\end{array}$ & $\begin{array}{l}\text { Todos os profissionais da APS realizam } \\
\text { busca ativa de casos novos para } \\
\text { identificação precoce, dose } \\
\text { supervisionada, a avaliação dos contatos, a } \\
\text { educação em saúde. A visita domiciliar é } \\
\text { uma tecnologia utilizada frequentemente } \\
\text { pela equipe para a realização das ações de } \\
\text { controle da hanseníase. }\end{array}$ \\
\hline $\begin{array}{l}\text { A13 (25)/ } \\
2016\end{array}$ & $\begin{array}{l}\text { Analisar a assistência de } \\
\text { enfermagem utilizada no } \\
\text { atendimento de portadores de } \\
\text { hanseníase. }\end{array}$ & $\begin{array}{l}\text { Estudo retrospectivo, } \\
\text { prospectivo, com } \\
\text { abordagem } \\
\text { quantitativa. }\end{array}$ & $\begin{array}{l}\text { Constatou-se a importância da assistência } \\
\text { de enfermagem na realização das ações de } \\
\text { controle da hanseníase no município, em } \\
\text { especial, o acompanhamento do } \\
\text { tratamento e a prevenção de } \\
\text { incapacidades. }\end{array}$ \\
\hline $\begin{array}{l}\text { A14 (26)/ } \\
2017\end{array}$ & $\begin{array}{l}\text { Conhecer a percepção dos } \\
\text { profissionais de saúde e gestores } \\
\text { sobre a atenção em hanseníase na } \\
\text { Estratégia Saúde da Família. }\end{array}$ & $\begin{array}{l}\text { Estudo descritivo } \\
\text { exploratório de } \\
\text { abordagem qualitativa. }\end{array}$ & $\begin{array}{l}\text { Educação em saúde para a comunidade é } \\
\text { realizada nas Equipes de Saúde da Família } \\
\text { por meio de intervenções em conjunto } \\
\text { com a equipe do Núcleo de Apoio à Saúde } \\
\text { da Família por meio de palestras e fixação } \\
\text { de cartazes informativos na unidade de } \\
\text { saúde. Houve treinamento dos ACS. }\end{array}$ \\
\hline
\end{tabular}

Após análise das variáveis, observou-se que os estudos foram realizados entre os anos de 2006 e 2017, não havendo nenhuma publicação nos anos 2010, 2012 e 2013. Nos demais anos, as publicações variaram entre uma $(7,1 \%)$ e duas $(14,3 \%)$. Em relação aos delineamentos mais encontrados, $64 \%$ dos estudos foram descritivos e $22 \%$ de estudos do tipo antes-depois com a realização de intervenção educativa.

Verificou-se que $11(78,7 \%)$ das publicações apresentavam dados brasileiros. As demais produções foram realizadas na Índia $(7,1 \%)$, em Mali $(7,1 \%)$ e em Cuba $(7,1 \%)$.

Após a leitura, análise e síntese dos resultados de cada estudo incluído na revisão integrativa, observou-se que as medidas preventivas para o controle da hanseníase 
poderiam ser agrupadas em categorias, que estão descritas na Tabela 2.

Tabela 2 - Categorias das medidas utilizadas na Atenção Primária à Saúde para o controle da hanseníase dos estudos incluídos na revisão integrativa.

\begin{tabular}{|c|c|c|c|}
\hline Categorias & Estudos & $\mathbf{n}$ & $\%$ \\
\hline 1) Educação em saúde para a comunidade & $\begin{array}{c}\mathrm{A} 2, \mathrm{~A} 4, \mathrm{~A} 8, \mathrm{~A} 10, \mathrm{~A} 12, \\
\mathrm{~A} 14\end{array}$ & 6 & 42,8 \\
\hline $\begin{array}{l}\text { 2) Educação em saúde para os profissionais } \\
\text { da APS }\end{array}$ & $\begin{array}{c}\mathrm{A} 1, \mathrm{~A} 2, \mathrm{~A} 3, \mathrm{~A} 6, \mathrm{~A} 9, \mathrm{~A} 11, \\
\mathrm{~A} 14\end{array}$ & 7 & 50,0 \\
\hline 3) Diagnóstico oportuno & $\mathrm{A} 1, \mathrm{~A} 2, \mathrm{~A} 4, \mathrm{~A} 9, \mathrm{~A} 11, \mathrm{~A} 12$ & 6 & 42,8 \\
\hline 3) Tratamento & A11, A12 & 2 & 14,3 \\
\hline 4) Vigilância dos contatos & $\mathrm{A} 4, \mathrm{~A} 9, \mathrm{~A} 11, \mathrm{~A} 12$ & 4 & 28,6 \\
\hline $\begin{array}{l}\text { 5) Atuação da enfermagem na vigilância } \\
\text { epidemiológica da hanseníase }\end{array}$ & $\mathrm{A} 7, \mathrm{~A} 13$ & 2 & 14,3 \\
\hline $\begin{array}{l}\text { 6) Descentralização das ações de prevenção } \\
\text { e controle para os serviços de APS }\end{array}$ & $\mathrm{A} 1, \mathrm{~A} 2, \mathrm{~A} 5, \mathrm{~A} 9$ & 4 & 28,6 \\
\hline
\end{tabular}

\section{Discussão}

Considerando que as medidas de controle devem ser desenvolvidas prioritariamente nas unidades da APS, e que são fundamentais para o alcance das metas estabelecidas pela OMS, foram identificadas as seguintes ações: capacitação profissional, descentralização das atividades, atividades de educação em saúde, definição de atribuições profissionais e uso de instrumentos específicos na assistência de Enfermagem.

Através das publicações, verificou-se que haviam achados satisfatórios quanto a capacitação profissional $(10,14,17)$ e acerca da realização de atividades de treinamento profissional nas modalidades presencial (16) e à distância (19). Foram descritas também, reuniões clínicas para discussão dos casos e esclarecimento de dúvidas (22).

Essas medidas vêm tendo sua importância demonstrada em instrumentos utilizados para avaliação em saúde, como o Primary Care Assessment Tool (PCAT) - versão brasileira, por meio do atributo orientação profissional, pois sabe-se que os profissionais possuem acesso aos treinamentos promovidos pelo Sistema Único de Saúde (SUS) e necessitam de formação específica para atuação na APS. Este atributo também faz parte do Instrumento de avaliação do desempenho da atenção primária nas ações de controle da hanseníase - versão profissionais - (PCAT - hanseníase) (27).

Estudos avaliativos desenvolvidos no Brasil evidenciaram que a formação profissional direcionada à hanseníase é heterogênea, principalmente quando avaliam as categorias profissionais de médicos e enfermeiros. Enquanto em Canaã dos Carajás (PA) os enfermeiros apresentam alta orientação para desenvolvimento de ações de controle de hanseníase (28), no mesmo município, os médicos avaliados demonstram uma baixa orientação (29). Isso demonstra a importância do incentivo à educação permanente, bem como sensibilização dos profissionais de saúde para a identificação e condução adequada dos casos de hanseníase.

As estratégias citadas nos estudos selecionados nessa revisão devem ser consideradas como prioritárias, pois diante do atendimento das necessidades de informação e atualização dos profissionais de saúde, eles poderão desempenhar uma assistência especializada à comunidade $(4,6)$. Para que treinamentos/ capacitações atinjam um número considerável de profissionais médicos, enfermeiros e demais categorias profissionais que atuam na APS, como os ACS que compõem as equipes de APS no Brasil, podem ser promovidas atividades presenciais e cursos à distância. 
Um dos estudos selecionados nesta revisão, descreveu um curso na modalidade à distância com o intuito de melhorar o diagnóstico precoce/ suspeita de hanseníase (19). Direcionado aos profissionais das equipes de Saúde da Família, o modelo interativo de tele-educação, demonstrou ser viável como recurso educacional para profissionais que atuam, principalmente, em regiões isoladas (19).

Outros exemplos de cursos à distância já foram implementados no Brasil: o curso Hanseníase na Atenção Básica, com carga horária de 45 horas, disponibilizado pela Universidade Aberta do SUS (UNASUS) (30) e o Curso de Capacitação em Ações de Eliminação da Hanseníase em Minas Gerais, em modalidade presencial (16 horas) e a distância (30 horas) (31).

As reuniões clínicas também são importantes para manter os profissionais da equipe mobilizados em relação à doença, tornando-os mais experientes, pois os casos discutidos são atividades de treinamento que direcionam a tomada de decisão e a qualidade do trabalho voltado à comunidade, contribuindo para efetivação da APS como porta de entrada do sistema de saúde e para maior resolubilidade (22).

Nesse sentido, inicia-se a abordagem das ações de controle onde foram mencionadas a descentralização das atividades e, ainda, as atividades assistenciais direcionadas aos pacientes de hanseníase e seus contatos para atender o objetivo de redução da carga da doença.

Os estudos referentes à descentralização das atividades de controle da hanseníase apresentam resultados de Duque de Caxias (Rio de Janeiro) (15) e do Estado do Paraná (18). Essa estratégia representa impactos positivos, pois contribui para um melhor acesso dos usuários às unidades de saúde, além de oportunizar diagnóstico oportuno e redução do estigma relacionado à doença $(1,4)$.

Embora os governos reafirmem a necessidade de implementação dessa estratégia, observa-se que, na APS, as ações de controle ainda são limitadas e que, a assistência ao paciente com hanseníase, na maioria das vezes, é centralizada nos serviços especializados (27). Para tanto, é necessário comprometimento de gestores em dar subsídios a APS e dos profissionais das ESF em reconhecer a hanseníase como importante problema de saúde pública (6).

Apesar de ser reconhecida pelos países endêmicos como importante, a descentralização não deve ser a única ação para o controle da doença. Fatores socioeconômicos, geográficos e políticos devem ser utilizados para planejar a aplicabilidade das medidas, como por exemplo a busca ativa de casos novos, a avaliação de contatos e a supervisão do tratamento (32).

Contudo, as evidências fortemente indicam que a estratégia prioritária para a redução da carga da hanseníase no mundo é a integração das ações de controle na APS (1). O desempenho dos serviços de APS na realização das ações de prevenção e controle da hanseníase pode ser facilmente avaliado com a utilização de instrumentos validados $(12,27,33,34)$ que conseguem medir a orientação desses serviços na implementação das atividades preconizadas pelo Ministério da Saúde (4).

Dessa forma, considera-se que a avaliação dos serviços de saúde, baseada em atributos orientadores, é capaz de caracterizar e qualificar os serviços de saúde, bem como gerenciar e reformular os sistemas de saúde ao evidenciar as potencialidades e fragilidades no desenvolvimento das ações (35).

Alguns estudos desta revisão, abordaram as atribuições dos profissionais da APS no controle da hanseníase e estão descritas como responsabilidade dos enfermeiros: a suspeita do diagnóstico, o tratamento adequado, avaliação de pacientes e contatos, acompanhamento do tratamento de reações, a prevenção de incapacidades e visitas domiciliares. Já as competências dos ACS envolvem a suspeição diagnóstica, o 
encaminhamento dos pacientes a UBS, busca ativa de contatos e faltosos e supervisão do tratamento (26).

Enfatiza-se que as atribuições dos profissionais da APS são baseadas em conhecimentos e práticas específicos que envolvem paciente e família, desde o diagnóstico à cura (5). Todas essas ações foram mencionadas, além da necessidade de reinserção social do paciente de hanseníase (26).

Frequentemente, a pessoa com hanseníase passa por exclusão social que se dá através de diversos fatores como pelo estigma da doença e pelo comprometimento das habilidades ocasionado pelas deformidades (36). Assim, entende-se que esses pacientes devem ser reinseridos em seus contextos laborais com adaptação de função ou conforme a sua condição de saúde permita e, também, nos contextos familiares e sociais, com a desmistificação da doença.

Também foi mencionado, como atribuição dos profissionais de nível superior, o treinamento dos ACS. Como estes são os profissionais que ligam a comunidade à equipe de saúde é imprescindível que sejam sensibilizados, qualificados e reconheçam as dificuldades do contexto da hanseníase, reconhecendo os sinais e sintomas sugestivos da doença durante as atividades na comunidade, principalmente durante as visitas domiciliares (37).

Nessa perspectiva, as atividades de educação em saúde, devem ser executadas amplamente na comunidade, pois tem o potencial de informar à população, esclarecer dúvidas e minimizar o preconceito em relação à doença. Conforme as recomendações, devem ser direcionadas às equipes de saúde, aos casos suspeitos e diagnosticados, aos contatos, aos líderes da comunidade e ao público em geral (4).

Nos estudos da revisão integrativa verificou-se que diferentes modalidades foram utilizadas, como uma intervenção educativa em Cuba direcionada aos contatos intradomiciliares a fim de melhorar seus conhecimentos em relação à hanseníase, por serem considerados uma população de risco. Após seleção dos participantes, estes responderam um questionário para avaliação do conhecimento sobre a doença. Posteriormente foram divididos em dois grupos e submetidos a intervenções semanais de uma hora, no período de um mês, onde eram realizadas apresentações, animações e análise das ações. Três meses após o último encontro, o questionário foi novamente aplicado e verificou-se melhora dos conhecimentos dos participantes (21).

Outra intervenção educativa foi desenvolvida em Uberaba, Minas Gerais, aplicado à população geral. Também identificou resultados satisfatórios, pois antes da intervenção educativa foi observada desinformação dos participantes acerca da hanseníase e após a ação, esses resultados melhoraram consideravelmente (38). Atividades como essas merecem ser disseminadas e incentivadas pelo potencial transformador de orientar os indivíduos sobre aspectos fundamentais da doença.

Também foram evidenciadas palestras mediadas pelos profissionais da equipe de Saúde da Família e do Núcleo de Apoio à Saúde da Família. No entanto, estavam descritas como pontuais por serem realizadas apenas por determinação dos programas governamentais implementados na APS (26). Dessa forma, entende-se que há baixa mobilização para o enfrentamento da doença que requer maiores investimentos para o alcance do fortalecimento das intervenções interinstitucionais e intersetoriais (40).

Faz-se importante que os profissionais de saúde utilizem os momentos de educação em saúde de maneira frequente, utilizando-se de atividades dinâmicas para promover uma maior interação com os usuários, para que estes participem ativamente esclarecendo dúvidas e relatando experiências.

Corroborando com a educação em saúde, as discussões em salas de espera são efetivas e também foram apontadas (24). Trata-se de uma estratégia que além de envolver os 
usuários, reduz as barreiras do conhecimento da doença, favorece a prevenção e possibilita o diagnóstico precoce. Destaca-se ainda que para potencializar os resultados promovidos pela iniciativa em sala de espera, os meios de comunicação devem ser integrados (38).

Nas descrições das publicações foram mencionadas a utilização de cartazes e distribuição de panfletos como abordagens frequentes nas unidades de saúde pois dão maior visibilidade a temática da hanseníase $(23,26)$. Embora sejam materiais educativos, ressalta-se que quando são usados de maneira verticalizada, pontual e instrumental podem dificultar a interação entre profissionais de saúde e usuários (39). Desse modo, a aplicação destes materiais deve promover uma mediação na relação entre profissionais da equipe e os usuários dos serviços (40).

Esses materiais devem ser periodicamente atualizados e distribuídos à comunidade com a expectativa de amplo alcance dos sujeitos (40). Para isto, é necessário que sejam facilmente compreendidos através de imagens e/ou linguagem apropriada, considerando as pessoas com baixa escolaridade ou que não sabem ler.

Outra ação que pode ser desenvolvida, considerando-se os meios de comunicação, são os programas de rádio, que podem intensificar as ações da vigilância epidemiológica e informar sobre ações de mobilização social. Além de ser um espaço adequado para disseminação de informações sobre diagnóstico, transmissão, cura e situação epidemiológica da doença nas diversas localidades, possibilita a troca de experiências e esclarecimento de dúvidas.

$\mathrm{Na}$ análise das publicações, também foram observados registros de consultas de enfermagem ao paciente com hanseníase (25) e à utilização de um instrumento de consulta de Enfermagem baseado no Processo de Enfermagem de Wanda Horta (20).

O enfermeiro integrante da equipe multiprofissional da APS deve atender os pacientes com hanseníase buscando o atendimento de suas necessidades e apoiando-os durante todo o processo de adesão ao tratamento e promoção do autocuidado (24). Durante as consultas de enfermagem é possível visualizar diversas ações de controle da hanseníase, principalmente as que orientam os pacientes acerca da doença, do tratamento e da prevenção de incapacidades.

Embora alguns desses estudos evidenciem o trabalho dos profissionais de enfermagem, ressalta-se que os cursos de graduação brasileiros não direcionam uma carga horária satisfatória para o ensino teórico-prático do manejo do paciente com hanseníase. O desenvolvimento de atividades extensionistas/ extracurriculares podem contribuir no desenvolvimento de competências e habilidades e melhor preparar esses profissionais para atuação nas ações de controle de hanseníase (37).

Observa-se ainda que a utilização de instrumentos próprios da assistência de Enfermagem agrupa informações para uma melhor compreensão do contexto biopsicossocial onde o indivíduo está inserido e, consequentemente, permite a elaboração de um plano de cuidados individualizado (20).

Apesar da compreensão de que esses instrumentos são necessários para orientação das ações de enfermagem à pessoa com hanseníase, o trabalho exercido por enfermeiros no Programa de Controle da Hanseníase em diversas instituições ainda não está sistematizado (41).

Assim, diante das ações preventivas e curativas direcionada aos indivíduos e familiares que devem ser implementadas pelos enfermeiros na APS, é fundamental que instrumentos de enfermagem possam ser aplicados com o intuito de identificar necessidades, definir intervenções e contribuir para a consolidação do cuidado profissional da enfermagem.

Entretanto, existem outras formas de sistematizar o atendimento a pessoa com 
hanseníase. Isto ocorre a partir do uso de protocolos, manuais e planos que permitem uma assistência baseada em evidências, não só pelo profissional enfermeiro, mas por todos os profissionais da APS, como médico, odontólogo, psicólogo, fisioterapeuta, ACS e outros (5). Se o município não possuir protocolo próprio para atendimento dos casos de hanseníase, é importante orientar aos profissionais a utilização dos manuais oficiais que dispõem sobre a vigilância e controle do agravo (6), por exemplo, o manual técnico operacional do Ministério da Saúde (4).

Foram definidas como limitações do estudo a ausência de estudos com delineamento de ensaios clínicos controlados e de coorte e a inclusão dos artigos disponíveis somente online e gratuitos.

\section{Conclusão}

As medidas de prevenção e controle descritos nos artigos consultados na revisão integrativa apontam consonância com as recomendações mundiais e do governo brasileiro para a redução da carga da hanseníase. Também assinalam os esforços para a descentralização dessas ações para os serviços de APS tendo em vista a necessidade de conformação da rede de atenção à hanseníase.

Para sustentabilidade das ações de controle na APS é necessário que os profissionais estejam sensibilizados. Isto pode iniciar-se na graduação, perpassando no dia a dia com a realização de cursos, reuniões para discussão de casos, análise epidemiológica dos dados e estabelecimento de uma rede de atenção que apoie as ações da APS com fluxos de referência e contrarreferência bem definidos.

Para que a redução da carga da doença realmente aconteça, as ações apontadas pelos estudos devem acontecer de maneira associada além de implementadas constantemente. Ademais, as ações realizadas devem ser disseminadas/publicadas, mesmo em formato de relato de experiência, como forma de incentivo e valorização das atividades da APS.

\section{Referências}

1. World Health Organization. Global Leprosy Strategy: Accelerating towards a leprosy-free world. (Period 2016-2020). Nova Délhi: OMS-SEARO, 2016. Available from: https://apps.who.int/iris/handle/10665/254907

2. World Health Organization. Global leprosy update, 2018: moving towards a leprosyfree world. Weekly epidemiological Record. 2019 [cited 2019 Sep 04]; 94(35/36):389-412. Available from: https://apps.who.int/iris/bitstream/handle/10665/326775/WER9435-36-en-fr.pdf?ua=1

3. Ribeiro MDA, Silva JCA, Oliveira SB. Estudo epidemiológico da hanseníase no Brasil: reflexão sobre as metas de eliminação. Rev Panam Salud Publica 2018; 42: e42.

4. Brasil. Ministério da Saúde. Diretrizes para vigilância, atenção e eliminação da Hanseníase como problema de saúde pública: manual técnico-operacional. Brasília: Ministério da Saúde, 2016. Disponível em: http://portalarquivos2.saude.gov.br/images/pdf/2016/fevereiro/04/diretrizeseliminacao-hanseniase-4fev16-web.pdf

5. Minas Gerais. Secretaria de Estado da Saúde. Plano de Enfrentamento da Hanseníase em Minas Gerais, 2019-2022/ Secretaria de Estado de Saúde de Minas Gerais. Coordenadoria Estadual de Controle da Hanseníase 2019. - Belo Horizonte: UFMG, 2019.

6. Sousa GS, Silva RLF, Xavier MB. Hanseníase e Atenção Primária à Saúde: uma avaliação de estrutura do programa. Saúde debate 2017; 41(112): 230-242.

7. Leano HAM, Araújo KMFA, Bueno IC, Niitsuma ENA, Lana FCF. Socioeconomic factors related to leprosy: an integrative literature review. Rev Bras Enferm 2019; 72(5): 1405-15.

8. Uwimana I, Bizimungu N, Ingabire F, Mukamukwiye E, Sharangabo O, Ngabonziza S, Kamanzi, E. Trends in leprosy case detection in Rwanda, 1995-2011: analysis of 17 years of laboratory data. African Journal of Laboratory Medicine 2017; 6(1): 1-4. 
9. Mantellini GG, Gonçalves A, Padovani CR. Políticas públicas referentes às incapacidades físicas em hanseníase na virada do século: uma década de (des)controle? Physis 2019; 29(1): e290105.

10. Carvalho APM, Fabri ACOC, Lanza FM, Lopes FN, Lana FCF. Integration of leprosy control activities from the perspective of health professionals. Rev enferm UFPE 2015; 9(1): 114-20.

11. Romanholo HSB, Souza EA, Ramos Júnior AN, Kaiser ACGCB, Silva IO, Brito AL et al. Vigilância de contatos intradomiciliares de hanseníase: perspectiva do usuário em município hiperendêmico. Rev Bras Enferm 2018; 71(1): 163-9.

12. Lanza FM, Vieira NF, Oliveira MMC, Lana FCF. Evaluation of the Primary Care in leprosy control: proposal of an instrument for users. Rev Esc Enferm USP 2014; 48(6): 1054-61.

13. Mendes KDS, Silveira RCCP, Galvão CM. Revisão integrativa: método de pesquisa para a incorporação de evidências na saúde e na enfermagem. Texto contexto - enferm. 2008; 17(4): 758-64.

14. Pandey A, Patel R, Uddin MJ. Leprosy control activities in India: integration into general health system. Lepr Rev 2006; 77: 210-18.

15 Cunha MD, Cavaliere FAM, Hércules FM, Duraes SMB, Oliveira MLW, Matos HJ. Os indicadores da hanseníase e as estratégias de eliminação da doença, em município endêmico do Estado do Rio de Janeiro, Brasil. Cad. Saúde Pública 2007; 23(5): 1187-97.

16. Faye O, Hay RJ, Ryan TJ, Keita S, Traore AK, Mahé A. A public health approach for leprosy detection based on a very short term-training of primary health care workers in basic dermatology. Lepr Ver 2007; 78(1): 11-6.

17. Pereira AJ, Helene LMF, Pedrazini ES, Martins CL, Vieira CSCA. Atenção básica de saúde e a assistência em Hanseníase em serviços de saúde de um município do Estado de São Paulo. Rev. bras. Enferm 2008; 61: 716-25.

18. Silva Sobrinho RA, Mathias TAF. Perspectivas de eliminação da hanseníase como problema de saúde pública no Estado do Paraná, Brasil. Cad. Saúde Pública. 2008; 24(2): 303-14.

19. Paixão MP, Miot HA, Souza PE, Haddad AE, Wen CL. A university extension course in leprosy: telemedicine in the Amazon for primary health care. Journal of Telemedicine and Telecare 2009; 15: 64-7.

20. Duarte MTC, Ayres JA, Simonetti JP. Consulta de enfermagem: estratégia de cuidado ao portador de hanseníase em atenção primária. Texto contexto - enferm 2009; 18(1): 100-7.

21. Griñán AED, Martínez MR, Reyes JC, Capdesuñer AS, Sigler LR. Modificación de conocimientos sobre lepra en la atención primaria de salud. MEDISAN 2011; 15(6): 745-753.

22. Lanza FM, Lana FCF, Carvalho APM, Davi RFL. Ações de controle da hanseníase: tecnologias desenvolvidas nos municípios do Vale do Jequitinhonha, Minas Gerais. R. Enferm. Cent. O. Min $2011 ; 1(2): 164-75$.

23. Lana FCF, Lanza FM, Carvalho APM, Tavares APN. O estigma em hanseníase e sua relação com as ações de controle. Rev Enferm UFSM 2014; 4(3): 556-65.

24. Rodrigues FF, Calou CGP, Leandro TA, Antezana FJ, Pinheiro AKB, Silva VM et al. Knowledge and practice of the nurse about leprosy: actions of control and elimination. Rev Bras Enferm 2015; 68(2): 297-304.

25. Silva LSR, Silva TM, Rocha JT, Andrade WG, Lessa EC, Correia NS. Nursing care for leprosy patients assisted by the family health program. Rev enferm UFPE 2016; 10(11): 4111-7.

26. Girão Neta OA, Arruda GMMS, Carvalho MMB, Gadelha RRM. Percepção dos profissionais de saúde e gestores sobre a atenção em hanseníase na Estratégia Saúde da Família. Rev Bras Promoç Saúde 2017; 30(2): 239-48.

27. Lanza FM, Vieira NF, Oliveira MMC, Lana FCF. Validação do instrumento de avaliação do desempenho da atenção primária nas ações de controle da hanseníase (PCAT - hanseníase): versão profissionais. HU Revista 2018; 44(3): 311-23.

28. Sousa GS, Silva RLF, Xavier MB. atributos da atenção primária em saúde no controle da hanseníase: ótica do enfermeiro. Rev baiana enferm 2017; 31(1): e17251.

29. Sousa GS, Silva RLF, Xavier MB. Hanseníase e atenção primária: um estudo avaliativo sob a ótica do médico. Rev. Salud Pública. 2018; 20(3):359-365.

30. Brasil. Ministério da Saúde. Hanseníase na Atenção Básica. [cited 2020 Jan 02]. Available from: https://www.unasus.gov.br/cursos/curso/45292

31. Leroy FS, Coelho ACO, Niitsuma ENA, Gomes FBF, Lanza FM, Ribeiro GC et al. Educação 
permanente em saúde: a experiência do uso da educação a distância na capacitação em ações de controle da hanseníase. Em Rede 2017; 4(1): 1-16.

32. Saltarelli RMF, Seixas DHT. Limites e possibilidades na atenção ao portador de hanseníase no âmbito da Estratégia Saúde da Família. Rev. APS 2016; 19(4): 613-22.

33. Lanza FM, Vieira NF, Oliveira MMC, Lana FCF. Instrumento para avaliação das ações de controle da hanseníase na Atenção Primária. Rev. bras. enferm 2014; 67(3): 339-346.

34. Lanza FM, Vieira NF, Oliveira MMC, Lana FCF. Avaliação das ações de hanseníase desenvolvidas na atenção primária: proposta de um instrumento para gestores. Rev Min Enferm 2014; 18(3): 598-605.

35. Ferreira VD, Oliveira JM, Maia MAC, Santos JS, Andrade RD, Machado GAB. Avaliação dos atributos da Atenção Primária à Saúde em um Município Mineiro. Esc Anna Nery 2016; 20(4): e20160104

36. Ribeiro MDA, Oliveira SB, Filgueiras MC. Pós-alta em hanseníase: uma revisão sobre qualidade de vida e conceito de cura. Saúde (Santa Maria) 2015; 41(1): 9-18.

37. Souza RG, Lanza FM, Souza RS. Sensibilização dos Agentes Comunitários de Saúde para a atuação nas ações prevenção e controle da hanseníase: relato de experiência. HU revista 2018; 44(3): 411-15.

38. Moreira AJ, Naves JM, Fernandes LFRM, Castro SS, Walsh IAP. Ação educativa sobre hanseníase na população usuária das unidades básicas de saúde de Uberaba-MG. Saúde Debate 2014; 38(101): 234-43.

39. Lopes FN, Lana FCF. Social participation in leprosy control: a challenge for health services. Rev enferm UERJ 2015; 23(2): 235-40.

40. Kelly-Santos A, Monteiro SS, Ribeiro APG. Collection of educational materials on Hansen's disease: a tool for memory and communicative practices. Interface - Comunic. Saude, Educ 2010; 14(32): 3751.

41. Silva AS, Baitelo TC, Fracolli LA. Primary Health Care Evaluation: the view of clients and professionals about the Family Health Strategy. Rev. Latino-Am. Enfermagem 2015; 23(5): 979-87. 